\title{
Photochemical rearrangements of diazirines and thermal rearrangements of carbenes ${ }^{\ddagger}$
}

\author{
Matthew S. Platz,* Haiyong Huang, Francis Ford and John Toscano \\ The Ohio State University, 100 W. 18th Avenue, Columbus, Ohio 43210, USA
}

\begin{abstract}
Trans-3-(2-tert-butylcyclopropyl)-3H-diazirine was decomposed both thermally and photochemically to give the anticipated ring-expanded 3-tertbutylcyclobutene product, along with azine and products of trapping by solvent. In the presence of tetramethylethylene (TME), a bicyclopropane adduct was formed The yield of 3-tert-butylcyclobutene product, however, is only very slightly (0-7\%) decreased upon increasing the concentration of TME. Thus there must be two product-forming pathways. One pathway is a relaxed carbene intermediate, the second with the excited singlet state of the diazirine precursor. It is posited that upon activation diazirines open to form a diazirinyl diradical which undergoes the following processes: a)ring closure to reform the diazirine, b)formation of the linear diazo compound, c)cleavage to form carbene and dinitrogen, d)rearrangement to cyclobutene in concert with nitrogen extrusion.
\end{abstract}

\section{Introduction}

In the 1960's it was discovered that the pyrolysis and photolysis of the diazirine precursors of alkyl and dialkylcarbenes give different mixtures of stable products (ref. 1).

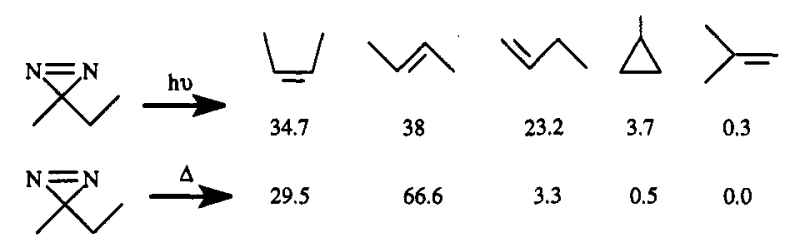

It was recognized that there were at least two routes to the formation of products. One was attributed to the relaxed carbene intermediate, and the other to vibrationally or electronically excited states of carbene or precursor. Other examples of this type were subsequently discovered (ref. 2).

In recent years, several laboratories, including our own, became interested in measuring the lifetimes of simple alkyl, dialkyl and cycloalkylcarbenes in fluid solution. These studies have demonstrated that simple carbenes are relatively long lived (ref. 3) and should have useful intermolecular chemistry in addition to rearrangement chemistry. It became apparent from the work of several groups (ref. 4) that intermolecular reactions should be much faster than intramolecular rearrangements. Nevertheless, it has been found that carbene traps can not completely suppress the yield of rearrangement products as predicted by laser flash photolysis studies. Again the need for two pathways from diazirine to rearrangement product becomes apparent.

Our reported laser flash photolysis studies of cyclopropylcarbene indicated that this carbene has a lifetime in hydrocarbon solution of $20 \mathrm{~ns}$ at ambient temperature which is controlled, at least in part, by reaction with solvent (ref. 5). Thus, cyclopropylcarbene should be sufficiently long lived, as is chlorocyclopropylcarbene, (ref. 6) to achieve bimolecular trapping chemistry in significant yields. This has prompted the present study of the bimolecular chemistry of trans-2-tertbutylcyclopropylcarbene. The tert -butyl group is present merely to raise the boiling point of precursor and products to facilitate their handling and detection.

Pyrolysis $\left(100^{\circ} \mathrm{C}\right)$ or photolysis $\left(350 \mathrm{~nm}, 25^{\circ} \mathrm{C}\right)$ of trans-3-(2-tert-butylcyclopropyl)-3H-diazirine 1 in $\mathrm{CF}_{2} \mathrm{ClCFCl}_{2}$ (Freon-113) induces the ring expansion reaction discovered in the parent cyclopropylcarbene system by Friedman and Shechter (ref. 7). Cyclobutene 2 is produced in 44$64 \%$ yield, along with a complex mixture of halogenated compounds and a small amount of tert- 
butylethylene 4 (Tables 1 and 2). When 1 is decomposed in Freon-113 containing 2,3-dimethyl2-butene (TME), adduct 3 is also produced in yields as high as 26-37\%. The yield of 3 smoothly increases as the concentration of TME increases (Tables 1 and 2).

TABLE 1. Absolute product yields in the pyrolysis of

1 in $\mathrm{CF}_{2} \mathrm{ClCFCl}_{2}\left(100^{\circ} \mathrm{C}\right)$ as a function of the concentration of TME.

\begin{tabular}{llll}
\hline $\operatorname{TME}(\mathrm{M})$ & \% 2 & \% 3 & \% 4 \\
\hline 0.0 & 64 & 0 & 14 \\
0.036 & 64 & 4.9 & 13 \\
0.067 & 65 & 8.7 & 12 \\
0.135 & 64 & 14 & 10 \\
0.261 & 64 & 20 & 8.4 \\
0.506 & 60 & 26 & 6.6 \\
0.948 & 57 & 32 & 3.9 \\
1.94 & 57 & 37 & 3.2 \\
\hline
\end{tabular}

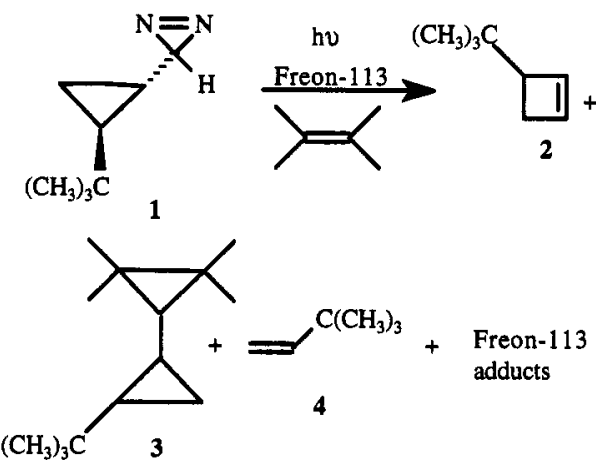

TABLE 2. Absolute product yields in the photolysis (350 nm) of 1 in $\mathrm{CF}_{2} \mathrm{ClCFCl}_{2}$

$\left(-25^{\circ} \mathrm{C}, 0^{\circ} \mathrm{C}\right.$ and $\left.25^{\circ} \mathrm{C}\right)$ as a function of the concentration of TME.

\begin{tabular}{llllllllll}
\hline $\operatorname{TME}(M)$ & $\% 2$ & $\mathbf{\% 3}^{-25} \mathrm{C}$ & $\% \mathbf{4}$ & $\% \mathbf{2}$ & $\begin{array}{l}0{ }^{\circ} \mathrm{C} \\
\mathbf{3}\end{array}$ & $\% 4$ & $\% 2$ & $\% 3$ & $\% 4$ \\
\hline 0.017 & 44 & 13 & 10 & 46 & 10 & 13 & 49 & 7 & 15 \\
0.034 & 47 & 19 & 10 & 44 & 14 & 12 & 46 & 10 & 14 \\
0.067 & 45 & 22 & 10 & 46 & 20 & 13 & 43 & 15 & 15 \\
0.134 & 46 & 26 & 11 & 48 & 24 & 13 & 47 & 22 & 13 \\
0.269 & 45 & 30 & 11 & 45 & 27 & 12 & 43 & 24 & 14 \\
0.420 & 46 & 32 & 10 & 47 & 28 & 13 & 46 & 27 & 13 \\
\hline
\end{tabular}

The critical observation is that the concentration of TME depresses the absolute yield of 2 formed on thermal $\left(100^{\circ} \mathrm{C}\right.$, Table 1$)$ or photochemical $\left(350 \mathrm{~nm}, 25^{\circ} \mathrm{C}\right.$, Table 2) decomposition of 1 by less than $7 \%$. At lower temperatures $\left(0^{\circ},-25^{\circ} \mathrm{C}\right)$ there is no decrease in the yield of cyclobutene $\mathbf{2}$ in the presence of TME. Therefore, 2 and $\mathbf{3}$ cannot issue from a common precursor. Again there must be two product-forming pathways. Similar results were obtained with n-propylamine. Unlike TME, this reagent will not undergo dipolar addition reactions with a putative diazo imtermediate and should not form an adduct without the intervention of a carbene.

Recently Moss et al (ref. 8) have reached the same conclusion with a closely related system, chlorocyclopropylcarbene 5 .<smiles>CC1(C)CC1CCl</smiles>

5

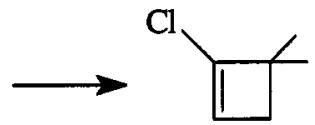

6

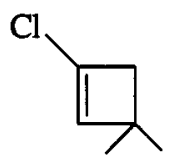

7

Similar observations that have been recently reported include benzylchloro (ref.9), methylchloro (ref.10), neopentylchloro (ref.11), cyclobutylchlorocarbene (ref.12), and cyclobutanylidene (ref.13). In all of these cases the alkene only incompletely intercepts the carbene of interest. These symtems all exhibit non-linear plots of the ratio of adduct/rearrangement versus trapping agent.

What are the two product-forming pathways? We postulate that upon pyrolysis or photolysis, diazirine 1 opens to form diradical 12. This process is well known in cyclopropene chemistry (ref. 14) and is predicted by theory (ref. 15) for the photochemical process.

This type of mechanism can also explain the original observations of Frey (ref. 1) and that of subsequent investigators, (ref. 2,4) who recognized the need for two product forming pathways. 

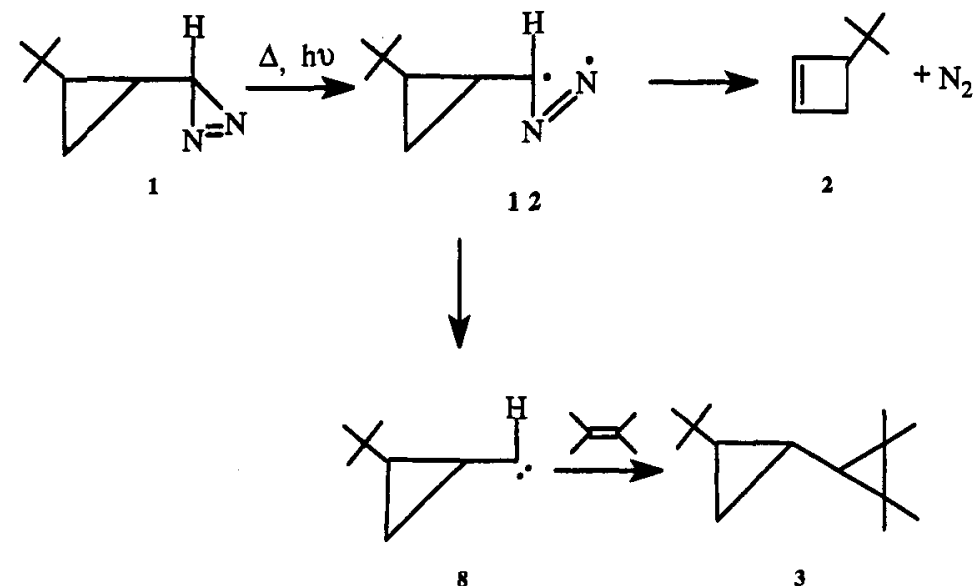

According to this mechanism, ring expansion of a cyclopropylcarbene, which is predicted to have an enthalpic barrier, can not compete in solution with faster intermolecular reactions of the carbene, which generally do not have an enthalpic barrier. Moss (ref. 6, 8) and Liu (ref. 16) were able to study the ring expansion of chlorocyclopropylcarbenes in solution because the chlorine substituent stabilizes the carbene and retards the rate of reaction of the carbene with solvent. In that case, the chlorocyclopropylcarbene is sufficiently long lived to undergo the ring expansion reaction.

Ring opening of a diazirine to a diradical is hardly a revolutionary idea. Certain azo compounds can fragment one $\mathrm{C}-\mathrm{N}$ bond at a time (ref. 17).

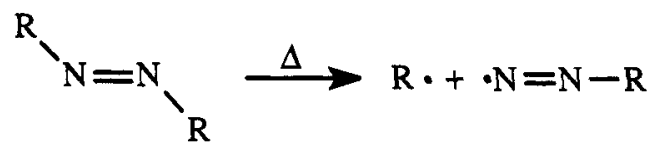

and cyclopropenes can ring open to form vinylcarbenes,
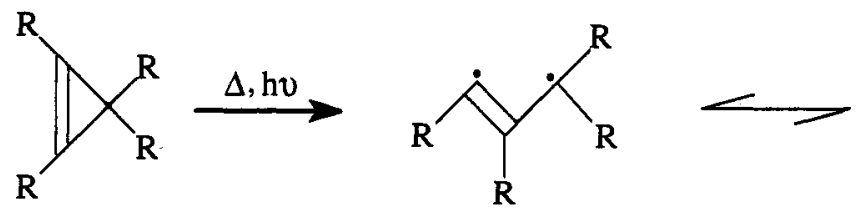<smiles>[R]CC([R])=C([R])[R]</smiles>

in addition to other processes (ref. 14b).

Our laboratory posits that the diradicals can suffer rearrangement in competition with the formation of diazirine and diazo compounds, and cleavage to carbene plus nitrogen. (Scheme 1)

Hydrogen migration to form ethylene in concert with nitrogen extrusion is certainly the most exothermic decay route available to diradical 13 (Scheme 1), formed from methyldiazirine or diazoethane. If this process is competitive with other, well known decay routes of 13 then the diradical can be the "second intermediate" needed to explain both the gas phase and solution phase data. It is important to note that the same diradical can also be formed thermally. It can also be formed upon activation of diazo precursors.

This decay route needs to be studied by ab initio theory to provide it with more justification then the present convenience of economy. Hopefully this will soon be forthcoming by masters of the art.

\section{Photophysical and Photochemical Evidence For Excited State Rearrangement}

Theorists and spectroscopists agree that the characteristic UV absorption of diazirines above 300 $\mathrm{nm}$ is due to an $\mathrm{n}$ to $\pi^{*}$ transition (ref. 15). This absorption is associated with a weak fluorescence (ref. 18,19 ). At $\approx 3 \mathrm{kcal} / \mathrm{mol}$ above the first excited state $\left(S_{1}\right)$ lies the $\mathrm{n}$ to $\sigma^{*}, \mathrm{~S}_{2}$ state.

Fragmentation of $S_{1}$ ( $n$ to $\pi^{*}$ ) leads to excited states of both methylene and nitrogen. It is the $S_{2}$ state ( $\mathrm{n}$ to $\sigma^{*}$ ) which correlates with ground state methylene and nitrogen and with 


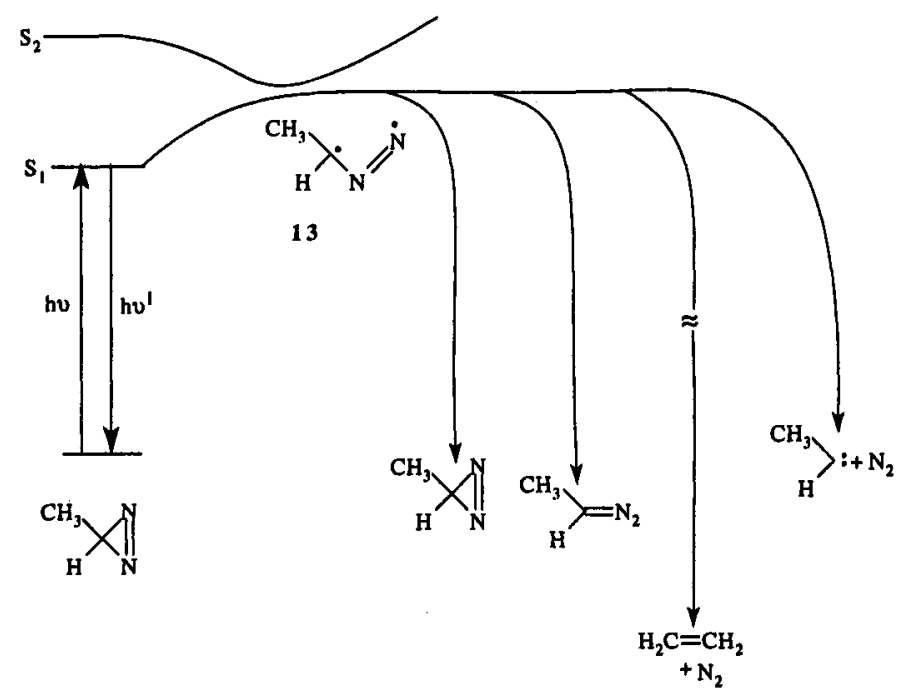

Scheme 1

isomerization to diazomethane. Thus, there must be an $n$ to $\sigma * n$ to $\pi^{*}$ conical intersection as explained by theory.

This picture explains the known temperature dependence of the fluorescence. (ref. 18, 19) The fluorescence intensities of dialkyldiazirines increase as the temperature decreases. This result demonstrates that the fluorescence of $S_{1}$ competes against an activated decay process. The apparent "activation energy" of this process is $2.8 \mathrm{kcal} / \mathrm{mol}$, the energy required to reach the $S_{1}, S_{2}$ surface crossing (ref. 15).

Spectroscopic evidence has been advanced in favor of the excited state rearrangement, by our laboratory, but we must admit that it is not overwhelming (ref. 18, 19).

According to our view, the fluorescence of diazirines is weak because it competes against other efficient excited state processes. Reducing the efficiency of the competitive processes by variation of diazirine structure should increase the fluorescence intensity. The observed order of fluorescence intensity is consistent with this prediction (ref. 20).<smiles>CCCC1N=NC12CC1CC3CC(C1)CC2C3</smiles><smiles>CC1CC1</smiles><smiles>CC1(C)N=N1</smiles>

Adamantyldiazirine and parent diazirine, which can not rearrange hydrogen in concert with nitrogen extrusion, fluoresce more brightly than does dimethyldiazirine which can rearrange.

The alert reader will realize that fluorescence proceeds from $S_{1}$, but that rearrangement transpires in a diradical formed after the $S_{1} / S_{2}$ surface crossing. This interpretation requires that the diradical must be able to reform the $S_{1}$ state in competition with hydrogen migration (Scheme 1).

The same explanation can explain the observed isotope effects on the fluorescence of dimethyldiazirine (DMD) and its perdeuterated isotopomer (DMD-d6). Modarelli et al. have reported that DMD-d6 is $1.55 \pm 0.20$ times as fluorescent as is DMD (ref. 18). Furthermore, DMD-d6 produces more trappable carbene than does DMD. Thus suppression of the excited state rearrangement by isotopic substitution improves the yield of the competitive processes e.g. fluorescence and cleavage to carbene.<smiles>CC1(C)N=N1</smiles>

DMD<smiles>CC1(C)N=N1</smiles>

DMD-d6 
Recently this work has been repeated and verified by Ford using more highly purified samples and a more sensitive fluorimeter (ref. 20 ).

It is also possible to explain the isotope effects as a consequence of enhanced internal conversion (IC) in DMD-d6 relative to DMD. However, Ford has performed the same type of experiments with diazirine and diazirine-d2. Surely IC will be greater in the smaller system. However no isotope effects on the yield of fluorescence or the yield of carbene are observed with diazirine and diazirine-d2 (ref. 20).<smiles>C1=NC1</smiles><smiles>[2H]C1([2H])N=N[C@@H]1P</smiles>

\section{References}

\# Portions of this work have been published previously.

H. Huang. and M. S. Platz. Tetrahedron Letters submitted

1. Platz, M S. Adv. Carbene Chem. II, Brinker U., ed., JAI Press, in press

D. R. Stevens. Tetrahedron Lett. 1733 (1966).

b)H. M. Frey and I. D. R. Stevens. J. Am. Chem. Soc. 87, 3101 (1965).

c)H. M. Frey. J. Am. Chem. Soc. 84, 2647 (1962).

d)H. M. Frey. Adv. Photochem. 4, 225 (1964).

e)L. Friedman and H. Shechter. J. Am. Chem. Soc. 81, 5512 (1959).

2. a)H. M. Frey and I. D. R. Stevens. J. Chem. Soc. 3101 (1965).

b)K. -T. Chang and . Shechter. J. Am. Chem. Soc. 101, 5082 (1979).

c)W. Kirmse and W. V. Wedel. Ann. Chem. 666, 1 (1963).

d)M. Fukushima, M. Jones, Jr. and U. Brinker. Tetrahedron Lett. 23, 2212 (1982).

e)J. M. Fox, J. E. G. Scacheri, K. G. L. Jones, M. Jones, Jr., P. B. Shevlin, B. Armstrong and R. Szytrbicka. Tetrahedron Lett. 33, 5021 (1992).

3. M. S. Platz, D. A. Modarelli, S. Morgan, W. R. White, M. Mullins, S. Celebi, J. P. Toscano and H. Huang. Prog. React. Kinetics 19, 93 (1994).

4. M. S. Platz. Advances in Carbene Chemistry II, U. Brinker, ed., JAI Press, in press.

5. D. A. Modarelli, M. S. Platz, R. S. Sheridan and J. R. Amman. J. Am. Chem. Soc. 115, 10440 (1993)

6. a)G. -J. Ho, K. Krough-Jespersen, R. A. Moss, S. Shen, R. S. Sheridan and R. Subramanian. J. Am. Chem. Soc. 111, 6875 (1989).

b)R. A. Moss, G. -J. Ho, S. Shen and K. Krough-Jespersen. J. Am. Chem. Soc. 112, 1638 (1990).

a)L. Friedman and H. Shechter. J. Am. Chem. Soc. 82, 1002 (1960).

b)J. A. Smith, H. Shechter, J. Bayless and L. Friedman. J. Am. Chem. Soc. 87, 935 (1965).

c)G. M. Kaufman, J. A. Smith, G. G. Vander Stoun and H. Shechter. J. Am. Chem. Soc. 87, 935 (1965).

8. R. A. Moss, W. Liu and K. Krough-Jespersen. J. Phys. Chem. 97, 13413 (1993).

9. a)H. Tomioka, N. Hayashi, Y. Izawa and M. -T.H. Liu. J. Am. Chem. Soc. 106, 454 (1984).

b)M. -T. H. Liu, N. Soundararajan, N. Paike, R. Subramanian. J. Org. Chem. 52, 4223 (1987).

10. J. A. LaVilla and J. L. Goodman. Tetrahedron Lett. 31, 5109 (1990).

11. R. A. Moss, G. -J. Ho, W. Liu and C. Sierakowski. Tetrahedron Lett. 34, 927 (1993).

12. R. A. Moss and G. -J. Ho. J. Phys. Org. Chem. 6, 126 (1993).

13. J. P. Pezacki, M. S. Platz and J. Warkentin. J. Am. Chem. Soc. submitted.

14. a)J. J. Gajewaski. Hydrocardon Thermal lsomerizations, pp. 22-25. Wiley, New York, N. Y. (1981).

b)I. Likhotvorik, D. W. Brown and M. Jones, Jr. J. Am. Chem. Soc. 116, 6175, (1994).

15. a)B. Bigot, R. Ponec, A. Sevin and A. V. Devaquet. J. Am. Chem. Soc. 100, 6575 (1978).

b)P. L. Müller-Remmers and K. Jug. J. Am. Chem. Soc. 107, 7275 (1985).

c)N. Yamamoto, F. Bernardi, A. Bottoni, M. Olivucci, M. A. Robb and S. Wilsey. J. Am. Chem. Soc. 116, 2064 (1994).

16. M. T. H. Liu and R. J. Bonneau. J. Phys. Chem. 93, 7298 (1989).

17. P. S. Engel. Chem. Rev. 80, 99 (1980).

18. D. A. Modarelli, S. Morgan, and M. S. Platz. J. Am. Chem. Soc. 114, 7034-7041 (1992).

19. J. Buterbaugh, J. P. Toscano, T. Gustafson and M. S. Platz. Unpublished research at The Ohio State University.

F. Ford and M. S. Platz. Unpublished research at The Ohio State University. 